EXTENDED REPORT

\title{
Decreased prevalence of atopy in paediatric patients with familial Mediterranean fever
}

\author{
C Sackesen, A Bakkaloglu, B E Sekerel, F Ozaltin, N Besbas, E Yilmaz, G Adalioglu, S Ozen
}

Ann Rheum Dis 2004;63:187-190. doi: 10.1136/ard.2003.007013

See end of article for authors' affiliations .....................

Correspondence to: Dr S Ozen, Hacettepe University Medical Faculty, Paediatric Nephrology and Rheumatic Diseases Unit, Sihhiye, 06100 Ankara, Turkey; sezaozen@hacettepe. edu.tr

Accepted 20 May 2003

Background: A number of inflammatory diseases, including familial Mediterranean fever (FMF), have been shown to be driven by a strongly dominated Th1 response, whereas the pathogenesis of atopic diseases is associated with a Th2 response.

Objective: Because dominance of interferon gamma has the potential of inhibiting Th2 type responsesthat is, development of allergic disorders, to investigate whether FMF, or mutations of the MEFV gene, have an effect on allergic diseases and atopy that are associated with an increased Th2 activity.

Method: Sixty children with FMF were questioned about allergic diseases such as asthma, allergic rhinitis, and atopic dermatitis, as were first degree relatives, using the ISAAC Study phase II questionnaire. The ISAAC Study phase II was performed in a similar ethnic group recruited from central Anatolia among 3041 children. The same skin prick test panel used for the ISAAC Study was used to investigate the presence of atopy in patients with FMF and included common allergens.

Results: The prevalences of doctor diagnosed asthma, allergic rhinitis, and eczema were 3.3, 1.7, and $3.3 \%$, respectively, in children with FMF, whereas the corresponding prevalences in the ISAAC study were $6.9,8.2$, and $2.2 \%$, respectively. Only the prevalence of allergic rhinitis was significantly different between the two groups $(p<0.001)$. The prevalence of atopy in these patients with FMF $(4 / 60(7 \%))$ was significantly lower than in the children of the population based study $(20.6 \%)(p<0.001)$.

Conclusion: Family Mediterranean fever seems to be protective against development of atopic sensitisation and allergic rhinitis.

$\mathrm{T}$ he allergic diseases are known to be mediated by T helper 2 (Th2) cells characterised by the production of interleukin 4 (IL4). The pathogenesis of asthma, allergic rhinitis, and atopic dermatitis depends on IL4 for the class switching of $\mathrm{B}$ cell responses to secrete IgE antibodies. Interferon gamma (IFN $\gamma)$, produced by Thl cells, is a proinflammatory cytokine which helps $\mathrm{B}$ cells to produce autoantibodies and promotes macrophage-rich inflammatory reactions while inhibiting IgE dependent, eosinophil-rich reactions. ${ }^{1}$ A number of inflammatory diseases have been shown to be driven by a strongly dominated Thl response, ${ }^{23}$ whereas the pathogenesis of atopic diseases is associated with a Th2 response. Patients with Thl disease such as adult rheumatoid arthritis (RA) have been shown to have decreased prevalence of atopic disorders. ${ }^{3}$

Familial Mediterranean fever (FMF) is characterised by episodic inflammatory reactions of serosal tissues such as the pleura, peritoneum, and synovium associated with fever. ${ }^{4}$ The gene responsible for FMF, entitled MEFV (Mediterranean fever), encodes a leucocyte and monocyte specific inflammatory regulator, and its mutations cause the autoinflammatory phenotype of FMF. ${ }^{67}$ Centola et al have shown that MEFV expression was increased by IFN $\gamma$ and that it may have a place in the Thl mediated response. ${ }^{7}$ Further to this study, our group has shown that IFN $\gamma$ production by lymphocytes was significantly increased in patients who carried MEFV mutations, suggesting a Thl predominance in FMF. $^{8}$ Furthermore, in clinical practice we have noted that patients with FMF seldom if ever have atopic diseases, which require a Th2 predominance (unpublished observations).

Because dominance of IFN $\gamma$ has the potential to inhibit Th2-type responses - that is, development of atopic disorders, many Thl associated conditions have been shown to have an impact on Th2 driven conditions. ${ }^{3}$ Thus we aimed at investigating whether FMF, or mutations of the MEFV gene, had an effect on allergic diseases and atopy, which is associated with an increased Th2 activity.

\section{MATERIAL AND METHOD \\ Study design}

Children with FMF were screened for allergic diseases and atopic sensitisation. The results were compared with those of an age matched population from the ISAAC Study (International Study of Asthma and Allergies in Childhood)..$^{9} 10$

The study was approved by the Hacettepe University ethics committee, and written informed consent was taken from all participant's parents.

\section{Study group}

Sixty children with FMF (aged 5-16 years) were included in the study. All patients were Turkish and had typical phenotypes for FMF with recurrent attacks of fever and peritonitis, and/or pleuritis, and/or arthritis, and a dramatic response to colchicine. All were diagnosed and followed up by the Unit of Paediatric Nephrology and Rheumatology of Hacettepe University. All patients were receiving regular colchicine treatment during recruitment and none had renal amyloidosis. The study patients were mainly from central Anatolia $(80 \%)$, where our centre is located. None had an attack during the time of sampling.

Abbreviations: CRP, C reactive protein; ESR, erythrocyte sedimentation rate; FMF, familial Mediterranean fever; IFN $\gamma$, interferon gamma; IL, interleukin; ISAAC, International Study of Asthma and Allergies in Childhood; MEFV, Mediterranean fever gene; RA, rheumatoid arthritis; Th1, T helper 1; Th2, T helper 2 
All patients were questioned about allergic diseases such as asthma, allergic rhinitis, and atopic dermatitis, as were first degree relatives, using the ISAAC Study phase II questionnaire, ${ }^{11} 12$ which has previously been validated within the Turkish population. Parents were asked if a doctor had ever diagnosed asthma, hay fever, or eczema in their child. Children were defined as having asthma or atopic dermatitis if the diagnosis was previously made by a doctor. This was also the case for the diagnosis of allergic rhinitis in the presence of documented atopy. The questionnaire included questions on the current wheezing and ever wheezing. Current wheezing was defined as wheezing occurring within the past 12 months.

In addition, the questionnaire included questions on the history of atopic diseases in parents and FMF in the first degree relatives. The number of siblings and several other potential confounding factors, such as environmental tobacco smoke, attendance at day care, indoor pets, and indoor humidity, were asked about.

All the face to face interviews and allergy investigation was performed by two doctors (CS, BES).

The ISAAC Study phase II was conducted among children in Ankara, located in central Anatolia. Our study was performed in a similar ethnic group recruited from central Anatolia. The ISAAC Study was performed among 3041 children aged between 9 and 11 years old, between 1999 and 2000 and aimed at determining the prevalence of the atopic diseases and the role of contributing factors. The skin prick tests were performed on all study subjects with 11 common allergens.

\section{Laboratory}

Levels of total serum IgE (Pharmacia, Sweden) were measured in all our patients with FMF and in a randomly selected subgroup of 350 from the ISAAC study population. Routine management of the patients with FMF included determination of erythrocyte sedimentation rate (ESR) and C reactive protein (CRP) in order to investigate the disease activity. MEFV (the Mediterranean fever gene) mutation analysis was available in 58 of the patients. The hot spot exon 10, which harbours 14 mutations including M694V, M680I, V726A, and M694I, was first analysed by denaturing gradient gel electrophoresis. According to the band pattern, subsequent analysis was done either by restriction endonuclease enzyme digestion or sequencing. Furthermore, E148Q in exon 2 was analysed by restriction endonuclease enzyme (BstNI) digestion. ${ }^{13}$

The same skin prick test panel used for the ISAAC Study was used to investigate the presence of atopy in patients with FMF and included common allergens of Dermatophagoides pteronyssinus, $D$ farinae, grass mix, weed mix, tree mix, Alternaria, Cladosporium, cockroach, cat dander, cow's milk, and egg yolk of standardised extracts (ALK, Denmark or Allergopharma, Germany). Children with a wheal reaction $\geqslant 3 \mathrm{~mm}$, after subtraction of the reaction from the negative control, to one or more of the allergen tested, were considered to be atopic.

\section{Statistical method}

Results were expressed as the mean (SEM) or percentages with $95 \%$ confidence interval (CI). Group comparisons were made using $t$ test for population proportion. Two sided $\mathrm{p}$ values $<0.05$ were considered significant. All analyses were performed using the SPSS 11.0 program.

\section{RESULTS}

Sixty children (36 boys, 24 girls) diagnosed as FMF with a mean (SEM) age of $11.8(0.4)$ years were included in the study. The mean (SEM) duration of the disease was $7.3(0.4)$
Table 1 Demographic characteristics of the patients

\begin{tabular}{lll}
\hline & FMF study & ISAAC study \\
\hline No & 60 & 3041 \\
Age (year)* & $11.8(0.4)$ & $9.1(0.5)$ \\
Boys/girls & $36 / 24$ & $1536 / 1505$ \\
Duration of FMF* (years) & $7.3(0.4)$ & - \\
\hline *Mean (SEM). & & \\
\hline
\end{tabular}

years (table 1). The usual, onset of symptoms of FMF had occurred between the ages of 1 and 2 years.

All the patients were receiving colchicine treatment with an attack free period of at least 6-12 months. The mean (SEM) of ESR and CRP levels of the patients were 18.0 (2.0) $\mathrm{mm} / \mathrm{lst} \mathrm{h}$ and 4.4 (0.7) $\mathrm{mg} / \mathrm{l}$, respectively. Mutation analysis showed homozygosity or compound heterozygote state in 50 of the tested patients. Among these 65\% carried the M694V allele.

The prevalences of doctor diagnosed asthma, allergic rhinitis, and eczema in FMF were 3.3, 1.7, and 3.3\%, respectively, whereas the prevalences achieved in the ISAAC study were $6.9,8.2$, and $2.2 \%$, respectively. Comparison of the prevalences showed that only the difference in the prevalence of allergic rhinitis between the two groups was significant $(\mathrm{p}<0.001)$ (table 2$)$.

The prevalences of current wheezing in this study and the population based study were $13.3 \%$ and $11.5 \%(p=0.58)$, respectively, whereas prevalences of wheezing history were $25 \%$ and $22.2 \%(p=0.46)$, respectively, without any statistical significance between the two groups.

The prevalence of atopy $(6.7 \%)$ in patients with FMF was significantly lower than in the children $(20.6 \%)$ of the population based study $(\mathrm{p}<0.001)$. The prevalence of allergic diseases among the parents of patients with FMF (6.7\%) was significantly lower than that in the population based study $(49.5 \%) \quad(p<0.001)$. The prevalences of asthma, allergic rhinitis, and atopic dermatitis in the parents of the children with FMF and the population based studied cases were 5\% $v$ $5.2 \% \quad(p=0.94), \quad 1.7 \% \quad v 33 \% \quad(p<0.001)$, and $0 \% \vee v 9.8 \%$ $(\mathrm{p}<0.001)$, respectively.

The mean (SEM) values of IgE in FMF and the population based study were 35.0 (5.3) kU/l and 43.9 (3.1) kU/l, respectively. The mean (SEM) percentage of eosinophils in the peripheral blood of patients with FMF was $2.1(0.2 \%)$ (min: 0.2 and max: 8.6). Unfortunately, the eosinophil count was not determined in the population based study (ISAAC).

\section{DISCUSSION}

FMF is the most common autoinflammatory disease throughout the world. The carrier rate is as high as $1 / 5$ in the ethnic groups that are most commonly affected. ${ }^{13}{ }^{14}$ The high prevalence of this mutation has led to speculations on the possible advantages of this state. In view of the decreased prevalence of asthma in carriers and patients, it has been speculated that FMF may confer protection against asthma. ${ }^{15}{ }^{16}$ A reduction in the prevalence of atopic diseases has also been clearly shown in other Thl associated diseases, including adult RA. ${ }^{3}$ In fact it has been suggested that patients with RA may benefit from Th2 activity.

Our clinical observations had suggested to us that allergic problems were rare in patients with FMF. There is no report that colchicine modifies the cutaneous responsiveness in skin prick tests ${ }^{17}$ and no report that it has a therapeutic effect on atopic dermatitis. Although the prevalence of asthma is not significantly different in patients with FMF, we can say that previous studies concluded that colchicine had no treatment effect on asthma. ${ }^{18}$ Because colchicine does not have an antiallergic action we do not believe that the decreased atopic 
Table 2 The results of the study group. The prevalences are given as percentages with $95 \%$ confidence intervals

\begin{tabular}{lccl}
\hline & $\begin{array}{l}\text { FMF study (\%) } \\
\text { (95\% Cl) }\end{array}$ & $\begin{array}{l}\text { ISAAC study (\%) } \\
\text { (95\% CI) }\end{array}$ & p Values \\
\hline Doctor diagnosed asthma & $3.3(1.2$ to 7.8$)$ & $6.9(5.9$ to 7.8$)$ & NS \\
Doctor diagnosed allergic rhinitis & $1.7(1.5$ to 4.9$)$ & $8.2(7.2$ to 9.1$)$ & $<0.001$ \\
Doctor diagnosed atopic dermatitis & $3.3(1.2$ to 7.8$)$ & $2.2(1.6$ to 2.7$)$ & NS \\
History of current wheezing & $13.3(4.6$ to 21.9$)$ & $11.5(10.3$ to 12.6$)$ & NS \\
History of ever wheezing & $25(13.9$ to 36$)$ & $22.2(20.7$ to 23.6$)$ & NS \\
Atopy & $6.7(0.32$ to 13$)$ & $20.6(19.1$ to 22$)$ & $<0.001$ \\
FMF in parents and siblings & $36.7(13)$ & - \\
Family history of allergic diseases & $6.7(0.32$ to 13$)$ & $49.5(47.7$ to 51.2$)$ & $<0.001$ \\
Asthma in parents & $5(0.5$ to 10$)$ & $5.2(4.4$ to 5.9$)$ & NS \\
Allergic rhinitis in parents & $1.7(-1.5$ to 4.9$)$ & $33(31.3$ to 34.6$)$ & $<0.001$ \\
Atopic dermatitis in parents & $0(-0.83$ to 0.83$)$ & $9.8(8.7$ to 10.8$)$ & $<0.001$ \\
Passive tobacco smoke & $63.3(51$ to 75$)$ & $64.2(62.4$ to 65.9$)$ & NS \\
Indoor humidity & $10(2.3$ to 17.6$)$ & $8.1(7.1$ to 9.0$)$ & NS \\
Total lgE (kU/l), mean (SEM) & $35.0(5.3)$ & $43.9(3.1)$ & NS \\
\hline NS, not significant. & & &
\end{tabular}

sensitisation was due to the treatment. The presented results show that personal atopy in the patients and allergic diseases in the parents, who were accepted as obligate carriers, were indeed lower. Atopy, influenced mainly by genetic predisposition, and allergic rhinitis were seen in low prevalence in patients with FMF. Although the prevalences of atopy and allergic rhinitis were low in children with FMF, the prevalences of asthma and atopic dermatitis were not statistically different.

Each of the atopic phenotypes is probably the result of a polygenic inheritance and a complex interaction between genes and environmental factors. Before our study three groups had already studied the prevalence of asthma in patients with FMF. In one study the prevalence of asthma was $2.96 \%$ but was only $0.92 \%$ among patients with FMF $(\mathrm{p}<0.005) .{ }^{15}$ In another study, asthma was absent in all patients with FMF. ${ }^{16}$ The third study suggested that there were no statistically significant differences in the prevalence of asthma between patients and their spouses or between parents and controls. ${ }^{14}$ In the presented patients, genetic components being a major determinant of FMF seemed to offer protection against atopy but not asthma and atopic dermatitis. Allergic rhinitis is a Th2 driven disease that is a classical manifestation of atopy and it was decreased in patients with FMF. We believe that asthma was not decreased in our study because it is not a purely Th2 driven disease. Asthma is a common and complex condition, with considerable heterogeneity both in phenotype and underlying pathophysiology. The disease may or may not manifest itself with atopy, and may be progressive, stable, or spontaneously remitting. Recent scientific trends also suggest that the Th2 hypothesis for asthma is too simplistic ${ }^{19}$ as it has been shown that there are increased circulating blood levels of IFN $\gamma$ in acute severe asthma ${ }^{20}$ and in supernatants of resting and stimulated bronchoalveolar lavage leucocytes of asthmatic patients. ${ }^{21}$ The complex pathophysiology of asthma may partially explain the differences in the relevant reports of its prevalence in FMF.

The atopic diseases are mainly mediated by Th2-type response and are associated with the production of proinflammatory cytokines, including IL4, IL5, IL6, IL9, IL10, and IL13. In turn, these cytokines stimulate production of IgE and induce tissue eosinophilia, which are characteristic of atopic allergic disorders. In our study, although not statistically significant, the lower levels of $\operatorname{IgE}$ and eosinophils in children with FMF were suggestive of a non-atopic status. It is interesting that the pyrin protein, encoded by MEFV, is expressed exclusively in monocytes, neutrophils, and eosinophils, ${ }^{4}$ though its role in eosinophils remains elusive.
In vitro studies by Centola et al have demonstrated that proinflammatory activators, including the Thl cytokine IFN $\gamma$, tumour necrosis factor, and lipopolysaccharide, up regulate monocyte MEFV expression. ${ }^{7}$ This study suggests that MEFV mediates a Thl responsive, negative feedback loop during proinflammatory activation of myeloid cells and that the pathophysiological features of FMF result from defects in this inhibitory activity. We have previously shown by FACS analysis that both patients with FMF and their parents had an increased Thl activity, expressed by the increased IFN $\gamma$ staining. ${ }^{8}$ IFN $\gamma$ is known to inhibit IgE dependent reactions. Thus the decreased atopy in people carrying mutations for the MEFV gene may simply be a secondary result of the Thl domination in these subjects. It is tempting to speculate that the trigger(s) that has selected the MEFV gene mutations may have upset the overall balance between the mutually antagonistic T helper cells to the advantage of the Thl cells. What the trigger is that led to such a marked selection remains obscure.

\section{Authors' affiliations}

C Sackesen, B E Sekerel, G Adalioglu, Hacettepe University Medical Faculty, Paediatric Allergy and Asthma Unit, Ankara, Turkey A Bakkaloglu, F Ozaltin, N Besbas, S Ozen, Hacettepe University Medical Faculty, Paediatric Nephrology and Rheumatic Diseases Unit, Ankara, Turkey

E Yilmaz, Hacettepe University Medical Faculty, Department of Molecular Biology, Ankara, Turkey

\section{REFERENCES}

1 Romagnoni S. Human Th1 and Th2 subsets. Doubt no more. Immunol Today 1991; 12:256-7.

2 Liblau RS, Singer SM, McDevitt HO. Th1 and Th2 CD4+T cells in the pathogenesis of organ-specific autoimmune diseases. Immunol Today 1995; 16:34-8.

3 van Roon JAG, Bijlsma JWJ. Th2 mediated regulation in RA and the spondyloarthropathies. Ann Rheum Dis 2002;61:951-4.

4 Ben-Chetrit E, Levy M. Familial Mediterranean fever. Lancet 1998;351:659-64.

5 Ozen S. Vasculopathy, Behçet's syndrome and familial Mediterranean fever. Curr Opin Rheumatol 1999;11:393-8.

6 The International FMF Consortium. Ancient missense mutations in a new member of the RoRet gene family are likely to cause familial Mediterranean fever. Cell 1997:90:797-807.

7 Centola M, Wood G, Frucht DM, Galon J, Aringer M, Farrell C, et al. The gene for familial Mediterranean fever, MEFV, is expressed in early leukocyte development and is regulated in response to inflammatory mediators. Blood 2000;95:3223-31.

8 Aypar E, Ozen S, Okur H, Kutluk T, Beșbass N, Bakkaloğlu A. Th1 polarization in familial Mediterranean fever. J Rheumatol 2003;30:201 1-13.

9 Saraclar Y, Kuyucu S, Tuncer A, Sekerel B, Sackesen C, Kocabas C. Prevalence of asthmatic phenotypes and bronchial hyperresponsiveness in Turkish schoolchildren: an International Study of Asthma and Allergies in 
Childhood (ISAAC) phase 2 study. Ann Allergy Asthma Immunol 2003;91:477-84

10 Kuyucu S, Saraclar Y, Tuncer A, Sackesen C, Adalioglu G, Sumbuloglu V, Sekerel BE. Determinants of atopic sensitization in Turkish school children: effects of pre- and post-natal events and maternal atopy. Pediatr Allergy Immunol (in press).

11 Asher MI, Weiland SK, on behalf of the ISAAC Steering Committee. The International Study of Asthma and Allergies in Childhood (ISAAC). Clin Exp Allergy 1998;28:S52-66.

12 The International Study of Asthma and Allergies in Childhood (ISAAC) Steering Committee. Worldwide variation in prevalence of symptoms of asthma, allergic rhinoconjunctivitis, and atopic eczema: ISAAC. Lancet 1998; 351:1225-32.

13 Yilmaz E, Ozen S, Balci B, Duzova A, Topaloglu R, Besbas N, et al. Mutation frequency of familial Mediterranean fever and evidence for a high carrier rate in the Turkish population. Eur J Hum Genet 2001;9:553-5.

14 Brenner-Ullman A, Melzer-Ofir H, Daniels M, Shohat M. Possible protection against asthma in heterozygotes for familial Mediterranean fever. Am J Med Genet 1994;53:172-5.
15 Danon YL, Laor A Shlezinger M, Zemer D. Decreased incidence of asthma in patients with familial Mediterranean fever. Isr J Med Sci 1990;26:459-60.

16 Ozyılkan E, Simsek H, Telatar H. Absence of asthma in patients with familial Mediterranean fever. Isr J Med Sci 1994;30:237-8.

17 Deanly P, Michel FB, Bousquet J. In vivo methods for study of allergy skin test techniques and interpretation. In: Middleton EJR, ed. Allergy principles, practice. St Louis, USA: Mosby, 1998:430-9.

18 Fish JE, Peters SP, Chambers CV, McGeady SJ, Epstein KR, Boushey HA, et al. An evaluation of colchicine as an alternative to inhaled corticosteroids in moderate asthma. Am J Respir Crit Care Med 1997;156:1165-71.

19 Salvi SS, Babu KS, Holgate ST. Is asthma really due to a polarized T cell response toward a helper T cell type 2 phenotype. Am J Respir Crit Care Med $2001 ; 164: 1343-6$

20 Corrigan $\mathrm{CJ}$, Kay AB. CD4+ T lymphocyte activation in acute severe asthma. Int Arch Allergy Immunol 1991;94:270-1.

21 Cembrzynska-Nowak M, Szklarz E, Inglot AD, Teodorczyk-Injeyan JA. Elevated release of tumor necrosis factor-alpha and interferon-gamma by bronchoalveolar leukocytes from patients with bronchial asthma. Am Rev Respir Dis 1993;147:291-5.

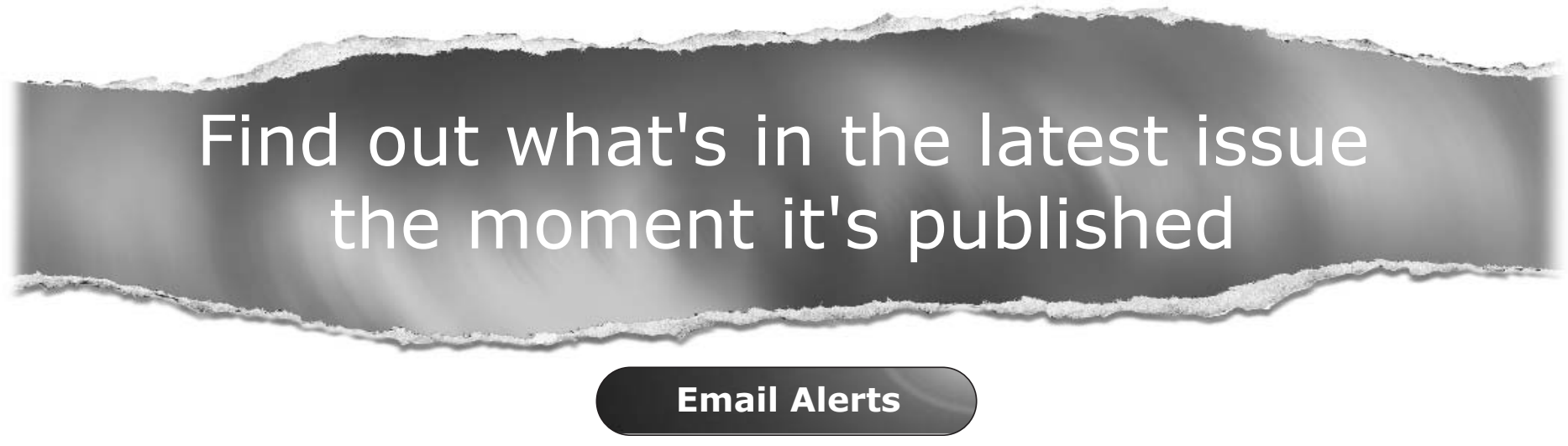

Sign up to receive the table of contents by email every month. You can select from three alerts:

Table of Contents (full), TOC Awareness (notice only); Annals of the Rheumatic Diseases related announcements.

\section{www.annrheumdis.com}

